\title{
Superior sulcus tumors with vertebral body involvement: A multimodality approach
}

\author{
William D. Bolton, MD, ${ }^{\mathrm{a}}$ David C. Rice, MD, ${ }^{\mathrm{a}}$ Adam Goodyear, MD, ${ }^{\mathrm{a}}$ Arlene M. Correa, PhD, ${ }^{\mathrm{a}}$ Jeremy Erasmus, MD, \\ Wayne Hofstetter, MD, ${ }^{\text {a }}$ Ritsuko Komaki, MD, ${ }^{\mathrm{c}}$ Reza Mehran, MD, ${ }^{\text {a }}$ Katherine Pisters, MD, Jack A. Roth, MD, ${ }^{\mathrm{d}}$ \\ Stephen G. Swisher, MD, ${ }^{a}$ Ara A. Vaporciyan, MD, ${ }^{a}$ Garrett L. Walsh, MD, ${ }^{a}$ Jason Weaver, MD, ${ }^{a}$ and Laurence Rhines, MD
}

Objective: Superior sulcus tumors with involvement of the spine have historically been considered unresectable. We have previously documented a 2-year survival of 54\% in patients treated with a multimodality approach. This work builds on our previous experience and examines the long-term outcomes.

Methods: A retrospective review was performed on patients with superior sulcus non-small cell lung cancer tumors with involvement of the vertebral column $(\mathrm{n}=39)$ treated at The University of Texas MD Anderson Cancer Center from 1990 to 2006. Their clinical and pathologic data were analyzed for short- and long-term outcomes.

Results: Group 1 included $8(21 \%)$ patients with neuroforamen or transverse process involvement, group 2 had $16(41 \%)$ patients with partial vertebrectomy, and group 3 had $15(38 \%)$ patients with total vertebrectomy. There were $2(5 \%)$ postoperative deaths, and $11(28 \%)$ patients had major complications. Margins were positive in 17 $(44 \%)$ patients. Recurrence occurred in $23(59 \%)$ patients and was local in $11(28 \%)$ patients, distant in 11 $(28 \%)$ patients, and both in $1(3 \%)$ patient. Median time to local recurrence was 7 months in patients with positive margins and has not been reached for patients with negative margins $(P=.007)$. Median, 2-year, and 5-year overall survival was 18 months, $47 \%$, and $27 \%$, respectively. On multivariate analysis, the only independent predictor of shorter survival was nodal metastases $(P=.001$; hazard ratio, 6.5; 95\% confidence interval, 2.2-19.2).

Conclusion: An aggressive multimodality approach involving surgical resection can be performed with acceptable morbidity in highly selected patients with superior sulcus tumors and vertebral invasion at a specialized center. Encouraging long-term survival can be achieved in patients with negative margins and no lymph node involvement.

Earn CME credits at

http://cme.ctsnetjournals.org

Superior sulcus tumors are a rare type of tumor that accounts for less than $5 \%$ of all cases of non-small cell lung cancer (NSCLC). ${ }^{1}$ They can be challenging to resect because of their location and involvement of surrounding structures, such as the brachial plexus, subclavian vessels, and spine. Invasion of the spine is a poor prognostic factor and is traditionally considered a contraindication for resection. Multimodality therapy, including surgical resection with preoperative or postoperative chemotherapy and radiation, is now considered optimal management. Because of the

\footnotetext{
From the Departments of Thoracic and Cardiovascular Surgery, ${ }^{\mathrm{a}}$ Diagnostic Radiology, ${ }^{\mathrm{b}}$ Radiation Oncology, ${ }^{\mathrm{c}}$ Thoracic Medical Oncology, ${ }^{\mathrm{d}}$ and Neurosurgery, ${ }^{\mathrm{e}}$ The University of Texas MD Anderson Cancer Center, Houston, Tex.

Read at the Eighty-eighth Annual Meeting of The American Association for Thoracic Surgery, San Diego, Calif, May 10-14, 2008.

Received for publication May 9, 2008; revisions received Nov 24, 2008; accepted for publication Jan 28, 2009.

Address for reprints: David C. Rice, MD, Department of Thoracic and Cardiovascular Surgery, The University of Texas MD Anderson Cancer Center, 1515 Holcombe Blvd, Box 445, Houston, TX 77030 (E-mail: drice@mdanderson.org).

J Thorac Cardiovasc Surg 2009;137:1379-87

$0022-5223 / \$ 36.00$

Copyright $(C) 2009$ by The American Association for Thoracic Surgery

doi:10.1016/j.jtcvs.2009.01.015
}

small numbers of patients with superior sulcus tumors and vertebral involvement, there have been no large studies that evaluate the optimal treatment algorithm for this highly selected group of patients. Nevertheless, several small retrospective series have demonstrated the feasibility of performing either partial or total vertebrectomy for select patients with vertebral invasion, and survival appears to be improved relative to that seen in historical control subjects. ${ }^{2-7}$ In 1999 , we reported a series of 17 patients who underwent resection of superior sulcus tumors that involved the spine. ${ }^{4}$ Overall 2 year survival was $54 \%$, but it was $80 \%$ in patients with negative margins. The purpose of this study was to update our previous experience by analyzing a larger number of patients and including data on long-term outcome.

\section{MATERIALS AND METHODS \\ Study Population}

The University of Texas MD Anderson Cancer Center Institutional Review Board granted approval for the study. Patients were identified by using the institutional tumor registry, as well as prospective patient databases maintained by the Departments of Thoracic and Cardiovascular Surgery and Neurosurgery. The study period was from January 1, 1990, to December 31, 2006. We defined a superior sulcus tumor as an apical NSCLC with radiographic, surgical, and/or pathologic involvement of the first rib or first intercostal space. For inclusion in the study, patients had to have either surgical or pathologic evidence of neuroforamen or vertebral invasion. Preoperatively, involvement of the spinal structures and staging of the primary tumor was evaluated by means of computed tomographic scan or magnetic 


\section{Abbreviation and Acronym \\ NSCLC $=$ non-small cell lung cancer}

resonance imaging in all patients. Nodal staging was performed with computed tomographic scanning, positron emission tomographic scanning, and mediastinoscopy $(n=7)$. Patients with tumors abutting the spine on preoperative imaging but found at the time of surgical intervention not to have vertebral or neuroforaminal invasion were excluded. Collected data included demographic information, preoperative imaging studies, clinical and pathologic staging, preoperative and postoperative therapy, extent of resection, margin status, perioperative data, and survival and recurrence information.

Fourteen $(36 \%)$ patients received preoperative chemotherapy, and 14 $(36 \%)$ received a preoperative radiation median dose of 46 Gy (range, 6$64 \mathrm{~Gy})$. Twelve (31\%) patients received both chemotherapy and radiation preoperatively. Chemotherapy was platinum based in all cases. Adjuvant chemotherapy regimens were similar and again all platinum based; however, higher doses of radiation were generally used (Table 1). Twenty-one $(54 \%)$ patients received postoperative radiation, with dose information available for 16 (median, $60 \mathrm{~Gy}$; range, 30-65 Gy). Eleven (28\%) patients received postoperative platinum-based chemotherapy. Of these, $8(21 \%)$ received both chemotherapy and radiation.

\section{Surgical Technique}

Extent of vertebral resection was classified as follows: group 1, patients with transverse process or neuroforamen involvement only; group 2, patients with vertebral body involvement requiring partial resection of one or more vertebrae; and group 3, patients with vertebral body involvement requiring complete resection of 1 or more vertebrae. In group 1 the transverse process was drilled out with a high-speed diamond burr power drill. Involvement of the surrounding posterior osseous elements was ablated with additional high-speed diamond burr resection. If there was significant extension of the tumor into the spinal canal or gross invasion of the proximal transverse process, facet joints, or lamina, a multilevel laminectomy was performed with a posterior midline extension of the thoracotomy incision. This allowed visualization of the thecal sac, as well as the ipsilateral nerve roots involved with the tumor. These roots could then be ligated proximal to the dorsal root ganglion. Patients in this group did not require spinal stabilization. In group 2 osseous involvement of the vertebral bodies was limited to the cortical bone, and partial vertebrectomy was performed with a highspeed diamond burr power drill and various sizes of curettes. The bony margin could not be assessed at the time of the operation, and therefore the resection was continued until, in the opinion of the attending neurosurgeon, grossly normal bone was visualized. The surrounding soft tissue structures were sent for frozen section analysis to ensure the tumor was not present at those margins.

Posterior stabilization was required in only 1 patient in this group. This patient had all the posterior elements, facet joints, and posterior aspects of the vertebral bodies at the 2 levels resected and was therefore believed to be unstable posteriorly. All patients in groups 1 and 2 had their spinal and lung resections performed as a single operative procedure. In group 3 extensive osseous involvement necessitated complete vertebrectomy. The decision to perform complete vertebrectomy was generally based on the amount of vertebral bony involvement demonstrated on preoperative imaging. Patients who had invasion beyond the cortex of the vertebra with significant cancellous involvement underwent total vertebrectomy. During the earlier part of the study, this was performed in the lateral decubitus position at the same time as lung resection by extending the thoracotomy incision posteriorly and cephalad to expose the spine. A multilevel laminectomy was performed followed by posterior instrumentation. After disarticulation of the involved ribs and chest wall resection, total vertebrectomy was
TABLE 1. Preoperative and postoperative chemotherapy regimens

\begin{tabular}{lllr}
\hline \multicolumn{1}{c}{ Preoperative } & & \multicolumn{1}{c}{ Postoperative } \\
\hline Cisplatnin/VP-16 & 3 & Cisplatnin/VP-16 & 4 \\
Cisplatnin/vinblastine & 2 & Cisplatnin/vinblastine & 1 \\
Cisplatnin/vinorelbine & 1 & Carboplatnin/taxol & 2 \\
Carboplatnin/gemcitabine & 1 & Carboplatnin/docetaxol & 1 \\
Carboplatnin/taxol & 7 & Adriamycin/Ifosfamide & 1 \\
& & Unknown & 2 \\
Summary & 14 & Summary & 11 \\
\hline
\end{tabular}

performed with an intralesional approach. The entire bony structure of the vertebrae was removed with either the high-speed drill or with mechanical debridement with a curette. Therefore no bony margin was left to analyze for margin status. The surrounding soft tissue structures were sent for frozen section analysis to ensure the tumor was not present at those margins.

The vertebrectomy defect was reconstructed with methylmethacrylate by using the chest tube technique described by Cooper, Errico, and their colleagues. ${ }^{89}$ Anterior fixation was obtained with an anterior cervical locking plate and screw construct. Since 2004, a 2-stage approach was used $(n=2)$. Multilevel laminectomy with posterior stabilization is performed first in the prone position, followed 24 or 48 hours later by thoracotomy and vertebral body resection. Expandable titanium cages (Figure 1) are used to reconstruct the vertebral column $(n=2)$. Surgical approach included cervicoanterior thoracotomy (Dartevelle technique; $\mathrm{n}=2$ ), posterolateral thoracotomy ( $\mathrm{n}$ $=28$ ), and combined thoracotomy $(\mathrm{n}=9)$. Where possible, we attempt to preserve the clavicle because its resection can result in severe shoulder deformity when combined with a posterolateral thoracotomy. ${ }^{10}$

\section{Statistical Analysis}

Statistical analysis was performed with SPSS software (version 15; SPSS, Inc, Chicago, Ill). Cox regression analysis and the Student's $t, \chi^{2}$, and Fisher's exact tests were used where appropriate. Survival was calculated from the date of surgical intervention until the date of last follow-up or death. Survival curves were estimated by using the Kaplan-Meier method with the log-rank analysis for survival comparisons. Local recurrence was defined as tumor recurrence within the operative bed, ipsilateral chest, or mediastinum. Time to recurrence was dated from the date of surgical intervention to first radiographic or pathologic confirmation of recurrent tumor.

\section{RESULTS \\ Patient Characteristics}

There were 39 patients, of whom $10(26 \%)$ were women, and the median age was 56 years (range, $35-75$ years). The most common presenting symptom was shoulder or arm pain, which was present in $36(92 \%)$ patients. Other presenting features included hand weakness in $14(36 \%)$ patients and Horner's syndrome in $11(28 \%)$ patients. On preoperative imaging, abutment or involvement of the subclavian vessels was present in $11(28 \%)$ patients, and $27(69 \%)$ patients had invasion of the brachial plexus (T1 nerve root in 20 patients and $\mathrm{T} 1$ and $\mathrm{C} 8$ nerve roots in 7 patients). Patient demographics and tumor characteristics are summarized in Table 2.

\section{Surgical Treatment}

Operations were performed with curative intent in 35 $(90 \%)$ patients and were palliative, for pain relief, or for 

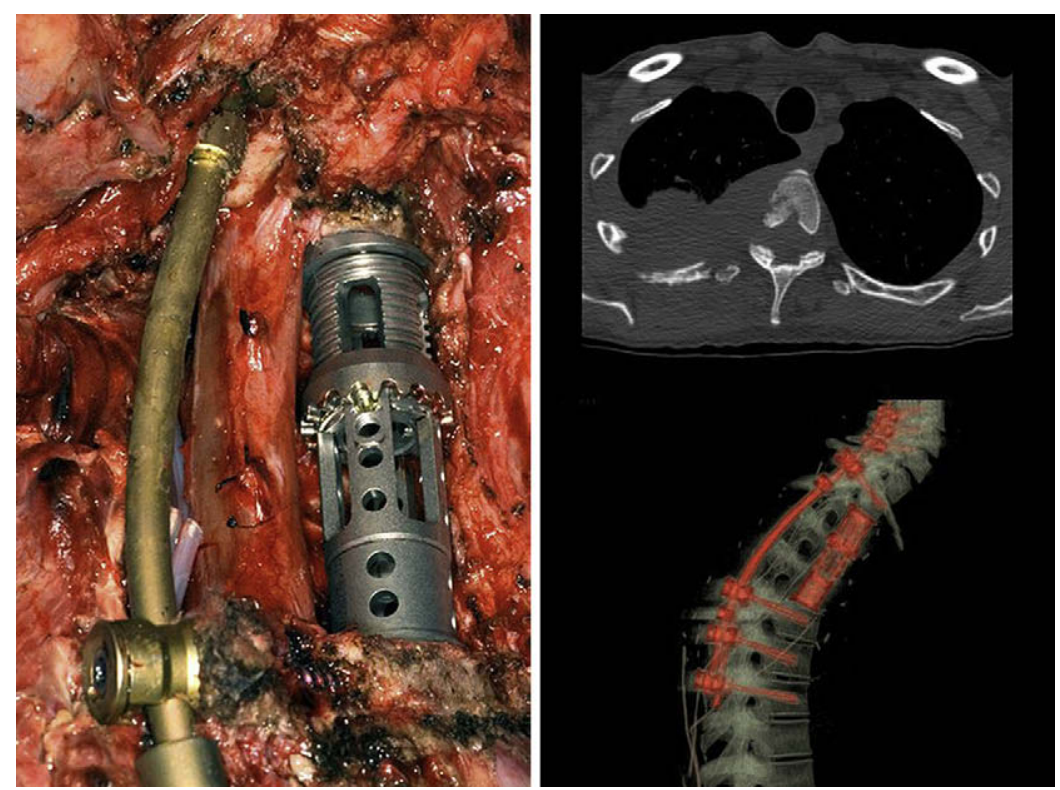

FIGURE 1. Patient with 3-level complete vertebrectomy with anterior and posterior stabilization. Currently, vertebral body reconstruction is performed with adjustable titanium cages (Synthes, Inc, West Chester, Pa), which are packed with bone graft.

prevention of spinal cord compromise in $4(10 \%)$ patients. Two $(5 \%)$ patients underwent resection through a cervicoanterior (Dartevelle technique) approach alone, with division of the manubrium and preservation of the ipsilateral clavicle. Nine $(23 \%)$ patients required combined cervicoanterior and posterolateral thoracotomy, and $28(72 \%)$ patients had posterolateral thoracotomy only. Although the tumor abutted the subclavian artery in $11(28 \%)$ patients, only 1 patient required subclavian artery resection. In this patient the subclavian artery was reconstructed with an 8-mm ringed polytetrafluoroethylene graft. The extent of pulmonary resection was decided based on the size and location of the lesion, and a wedge resection was performed at the discretion of the attending surgeon. This was based on the pulmonary status of the patient, the extent of lung involved, and the knowledge that recurrence typically occurs locally at the site of the vertebral resection or distant rather than within the pulmonary parenchyma or nodal basins.

Pulmonary resection included lobectomy in $26(67 \%)$ patients, pneumonectomy in $2(5 \%)$ patients, and wedge resection in $11(28 \%$ ) patients (Table 2). Wedge resections were equally distributed among all 3 groups in this study. Chest wall resection of 3 or more ribs was required in 30 $(77 \%)$ patients. Eight $(21 \%)$ patients had resection limited to the neuroforamen $(\mathrm{n}=1)$, transverse process $(\mathrm{n}=1)$, or both $(\mathrm{n}=6$; group 1$) ; 16(41 \%)$ patients required partial vertebrectomy (group 2); and $15(39 \%)$ patients had total vertebrectomy (group 3). In group 3 the median number of vertebral levels resected was 2 (Table 2). Three $(38 \%$ ) patients in group 1, $6(38 \%)$ in group 2, and $8(53 \%)$ in group 3 had positive surgical margins on final pathology $(P=.70)$.
The median length of stay was 10.5 days (range, 4-48 days). Major postoperative complications occurred in 11 $(28 \%)$ patients and included pneumonia $(\mathrm{n}=8)$, respiratory failure $(\mathrm{n}=5)$, pneumonitis $(\mathrm{n}=1)$, aspiration $(\mathrm{n}=1)$, empyema $(n=1)$, or death $(n=2)$. There were $2(5 \%)$ perioperative deaths, 1 from herpetic pneumonia in a patient after pneumonectomy and 2-level vertebrectomy and another from pneumonia and respiratory failure in a patient who had lobectomy and partial vertebrectomy.

\section{Pathologic Factors}

The median maximal tumor size was $5.9 \mathrm{~cm}$ (range, 2-24 $\mathrm{cm}$ ), and squamous cell carcinoma and adenocarcinoma accounted for $36 \%$ and $33 \%$ of cases, respectively (Table 2 ). Fourteen $(36 \%)$ patients had lymph node metastases. Of these, $5(13 \%)$ patients were N1, $6(15 \%)$ were $\mathrm{N} 2$, and 3 $(8 \%)$ had supraclavicular nodes (N3) involved. Complete resection (R0) was obtained in $22(56 \%)$ patients. Microscopic positive margins (R1) were found on the final pathologic specimen in the remaining $16(41 \%)$ patients. One patient had resection with residual macroscopic tumor remaining (R2).

\section{Nonsurgical Therapy}

One $(6 \%)$ patient who received preoperative treatment had a complete pathologic response. Two (13\%) patients had microscopic residual disease identified at surgical intervention, and the remaining $13(81 \%)$ patients had gross disease present at the time of the operation. Preoperative treatment with radiation, chemotherapy, or a combination of both did not have an effect on the completeness of resection (positive 
TABLE 2. Demographics

\begin{tabular}{|c|c|}
\hline Factor & $\mathbf{N}$ \\
\hline Patients & 39 \\
\hline Age, y (median [range]) & $56(35-75)$ \\
\hline \multicolumn{2}{|l|}{ Sex } \\
\hline Male & $29(74 \%)$ \\
\hline Female & $10(26 \%)$ \\
\hline \multicolumn{2}{|l|}{ Histology } \\
\hline Adenocarcinoma & $13(33 \%)$ \\
\hline Squamous & $14(36 \%)$ \\
\hline Large cell & $2(5 \%)$ \\
\hline Other* & $10(26 \%)$ \\
\hline \multicolumn{2}{|l|}{ Nodal status } \\
\hline N0 & $25(64 \%)$ \\
\hline N1 & $5(13 \%)$ \\
\hline $\mathrm{N} 2$ & $6(15 \%)$ \\
\hline N3 & $3(8 \%)$ \\
\hline \multicolumn{2}{|l|}{ Type of lung resection } \\
\hline Wedge & $11(28 \%)$ \\
\hline Lobectomy & $26(67 \%)$ \\
\hline Pneumonectomy & $2(5 \%)$ \\
\hline \multicolumn{2}{|l|}{ Type of vertebral resection } \\
\hline Transverse process or neuroforamen & $8(21 \%)$ \\
\hline Partial vertebrectomy & $16(41 \%)$ \\
\hline Total vertebrectomy & $15(38 \%)$ \\
\hline \multicolumn{2}{|l|}{ No. of vertebrae resected } \\
\hline 1 & $13(42 \%)$ \\
\hline 2 & $14(45 \%)$ \\
\hline 3 & $4(13 \%)$ \\
\hline \multicolumn{2}{|l|}{ Margin status } \\
\hline Positive & $17(44 \%)$ \\
\hline Negative & $22(56 \%)$ \\
\hline
\end{tabular}

*Sarcomatoid, poorly differentiated, or non-small cell lung cancer not otherwise specified.

margin). Recurrence and survival were similar for patients who received neoadjuvant treatment, adjuvant treatment, or both.

\section{Survival and Recurrence}

Median, 2-year, and 5-year overall survival was 18 months, $47 \%$, and $27 \%$, respectively (Figure 2). There was a nonsignificant trend toward decreased survival with increasing extent of vertebral resection (Table 3). Nodal metastases and positive resection margins were associated with worse survival (Table 3 and Figures 3 and 4). Multivariate analysis included the following variables: age, sex, tumor size, type of lung resection, preoperative chemotherapy, preoperative radiation, postoperative radiation, vertebral resection group, lymph node status, and margin status. Only lymph node status independently predicted survival $(P=.001$; hazard ratio, 6.5; $95 \%$ confidence interval, $2.2-19.2)$.

Tumor recurrence occurred in $23(59 \%)$ patients and was local in $11(28 \%)$ patients, distant in $11(28 \%)$ patients, and both local and distant in $1(3 \%)$ patient. Extent of vertebrectomy did not influence rates of local or distant failure or time

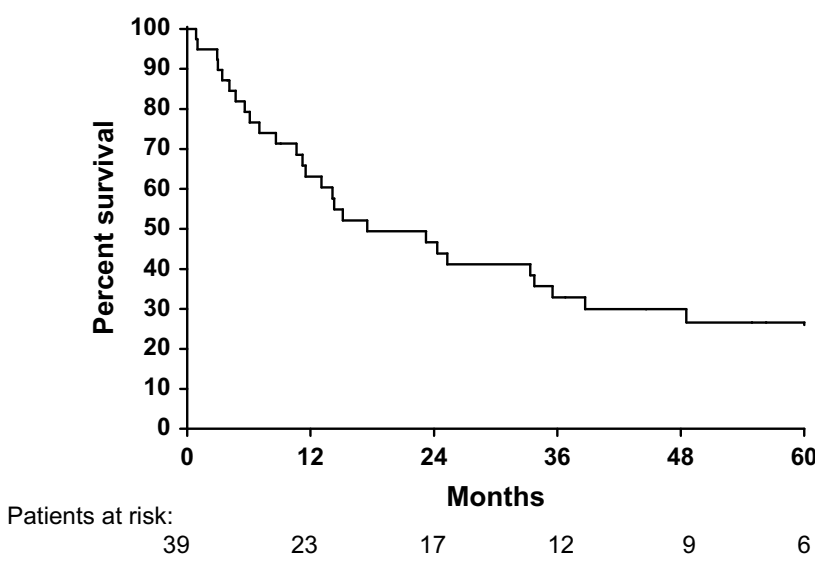

FIGURE 2. Overall survival from date of surgical intervention.

to recurrence. Twelve $(55 \%)$ patients with negative nodes had recurrence compared with $11(79 \%)$ patients with positive nodes $(P=.06)$. Median time to recurrence was significantly shorter in patients with positive nodes (5 vs 34 months, $P<.01)$. Patients with positive nodes had a higher incidence of local recurrence compared with patients with negative nodes $(57 \%$ vs $16 \%, P<.01)$; however, there was no difference in the rates of distant failure $(29 \%$ vs $24 \%, P=.52$ ). Recurrence was more frequent among patients with positive surgical margins $(76 \%$ vs $45 \%, P=$ .05 ), and time to first recurrence was also shorter (6.5 vs 23.9 months, $P<.05$ ). Local recurrence rates were higher in patients with positive margins $(53 \%$ vs $14 \%, P<.01)$, but there was no difference in the rates of distant failure $(29 \%$ vs $32 \%, P=.54)$. Multivariate analysis for overall recurrence included the following variables: type of lung resection, tumor size, preoperative chemotherapy, extent of vertebrectomy, lymph node status, and margin status. Only lymph node status independently predicted recurrence $(P=.001$; hazard ratio, $6.9 ; 95 \%$ confidence interval, $2.3-$ 20.9).

\section{DISCUSSION}

Superior sulcus tumors were first described by Pancoast ${ }^{11}$ in 1924, who postulated that the tumor originated from epithelial rests of the fifth branchial cleft. A bronchopulmonary origin was determined by Tobias ${ }^{12}$ in 1932 . These tumors

TABLE 3. Survival by group and nodal and margin status

\begin{tabular}{lrcrrc}
\hline & $\mathbf{n}$ & Median (mo) & $\mathbf{2} \mathbf{y}$ & $\mathbf{5} \mathbf{y}$ & $\boldsymbol{P}$ value \\
\hline Group 1 & 8 & 35.5 & $63 \%$ & $38 \%$ & .31 \\
Group 2 & 16 & 24.3 & $55 \%$ & $27 \%$ & \\
Group 3 & 15 & 11.2 & $29 \%$ & $22 \%$ & \\
Node negative & 25 & 38.7 & $71 \%$ & $41 \%$ & $<.001$ \\
Node positive & 14 & 7.0 & $0 \%$ & $0 \%$ & \\
Margin negative & 22 & 38.7 & $62 \%$ & $39 \%$ & $<.03$ \\
Margin positive & 17 & 13.1 & $29 \%$ & $12 \%$ & \\
\hline
\end{tabular}




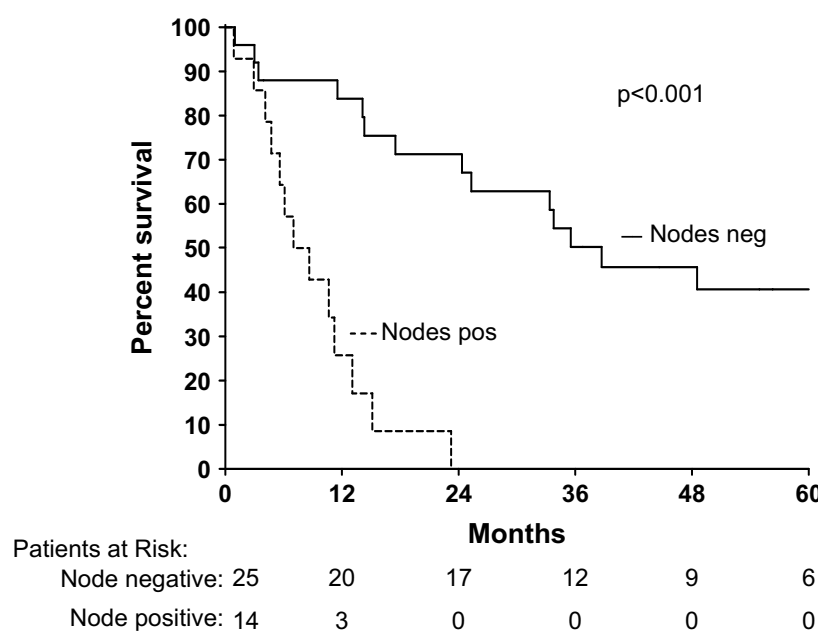

FIGURE 3. Survival by lymph node status. Solid line, negative nodes; dashed line, positive nodes.

are uncommon, and their location allows invasion into structures that can be difficult to resect, such as the vertebral bodies, brachial plexus, and subclavian vessels. These factors contributed to the original assumption that superior sulcus tumors were uniformly fatal. Over the last 2 decades, innovative surgical techniques have been developed that have allowed more extensive resections for lesions that were previously believed to be unresectable (ie, subclavian vessels, brachial plexus, and vertebral bodies). ${ }^{13,14}$

Several authors have described techniques for resection of lung cancer with invasion of the spine..$^{2-4,14,15}$ Our initial report included 17 patients with superior sulcus tumors that had spinal involvement. ${ }^{4}$ Of these, $14(82 \%)$ patients had vertebral body involvement, which required partial vertebrectomy in $7(50 \%)$ patients and total vertebrectomy in 7 $(50 \%)$ patients. As with the current series, en bloc resection was not performed, but rather high-speed diamond burr drills were used to perform intralesional resection of the involved vertebrae. There was no perioperative mortality; however, major complications occurred in $42 \%$ of patients. Despite the study sample consisting of a group of patients with advanced stage (T4) NSCLC, median overall survival was 25 months, and 2-year survival of patients with microscopically negative margins $(\mathrm{n}=11)$ was $80 \%$. This study showed that an aggressive surgical strategy that combined thoracic and neurosurgical techniques could result in better than expected survival and tumor control in a group of patients previously considered unresectable.

Our current study builds on our previous experience but includes larger patient numbers and longer follow-up. In common with our earlier report, the majority of patients had vertebral body involvement $(79 \%)$, and the proportions of patients undergoing partial and total vertebrectomy were similar $(52 \%$ and $48 \%$, respectively). Despite the fact that positive margins were more frequent in the current

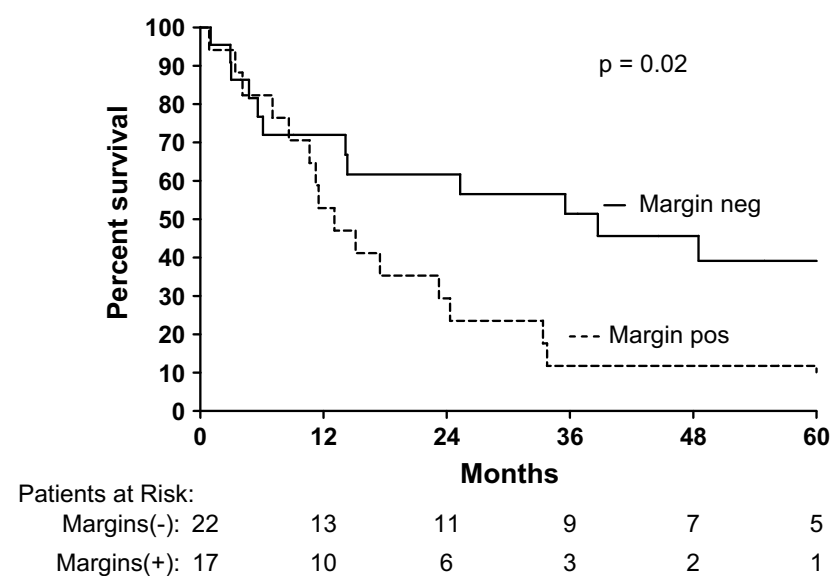

FIGURE 4. Survival by margin status. Solid line, Negative margins; dashed line, positive margin.

study ( $44 \%$ vs $35 \%$ ), local failure was slightly less common $(31 \%$ vs $41 \%)$, and distant failure was more frequent $(31 \%$ vs $12 \%$ ), perhaps related to a longer follow-up period in the current study. Similar to Gandhi and colleagues, ${ }^{4}$ we found that margin status significantly influenced not only local recurrence but also survival. However, at variance with our earlier report, we found that nodal metastases were an independent predictor of shorter survival. Interestingly, nodal metastases also were associated with local but not distant recurrence.

Several studies published in the English-language literature address surgical management of this specific population of patients. A series of 42 patients with superior sulcus tumors with spinal or brachial plexus involvement from Memorial Sloan Kettering Cancer Center were reported by Bilsky and associates. ${ }^{15}$ Of these, vertebral invasion (anterior or posterior elements) was present in only $14(33 \%)$ patients, with periosteal or neuroforaminal involvement present in $38 \%$ and $29 \%$, respectively. This contrasts with our current study, in which vertebral body invasion was present in $31(79 \%)$ patients. Bilsky and associates performed vertebrectomy in a manner similar to our approach with intralesional resection, and although the exact number of patients undergoing vertebrectomy was not reported, overall survival (17 months) was remarkably similar to that seen in our current study (18 months). Despite more patients having received neoadjuvant treatment in the Memorial series compared with ours $(66 \%$ vs $41 \%)$ and fewer patients with vertebral invasion, rates of local recurrence and survival were almost identical (Table 4).

Another study, by Grunenwald and coworkers, ${ }^{5}$ reported 19 patients who underwent surgical intervention for lung cancers that involved the spine. It is important to note that this study included patients with superior sulcus and nonsuperior sulcus tumors. As in our study, the majority of patients had vertebral invasion $(68 \%)$; however, the 
TABLE 4. Comparison table between the current study and those by Bilsky et $\mathrm{al}^{15}$ and Grunenwald et $\mathrm{al}^{5}$

\begin{tabular}{lccc}
\hline & Bilsky et al $\mathbf{l}^{\mathbf{1 5}}$ & Grunenwald et al $\mathbf{5}^{\mathbf{2}}$ & Current study \\
\hline No. & 42 & 19 & 39 \\
Vertebral body invasion & $14(33 \%)$ & $13(68 \%)$ & $31(79 \%)$ \\
Node positive & $\mathrm{NR}$ & $6(32 \%)$ & $14(36 \%)$ \\
En bloc & $\mathrm{No}$ & Yes & No \\
Partial vertebrectomy & $\mathrm{NR}$ & $15(79 \%)$ & $16(52 \%)$ \\
Total vertebrectomy & $\mathrm{NR}$ & $4(21 \%)$ & $15(48 \%)$ \\
R0/R1 (complete) & $27(64 \%)$ & $15(79 \%)$ & $22(56 \%)(\mathrm{R} 0)$ \\
R2 (incomplete) & $15(36 \%)$ & $4(21 \%)$ & $17(44 \%)(\mathrm{R} 1 / \mathrm{R} 2)$ \\
Neoadjuvant & $28(66 \%)$ & $11(58 \%)$ & $16(41 \%)$ \\
Adjuvant & $9(21 \%)$ & $5(26 \%)$ & $24(62 \%)$ \\
Major complications & $14(33 \%)$ & $9(47 \%)$ & $11(28 \%)$ \\
Length of stay & $\mathrm{NR}$ & $30 \mathrm{days}$ & $10 \mathrm{days}$ \\
Median survival & $17.3 \mathrm{mo}$ & $24 \mathrm{mo}$ & $18 \mathrm{mo}$ \\
2-y Survival & $44.00 \%$ & $53 \%$ & $47 \%$ \\
5-y Survival & $26 \%$ & $14 \%$ & $27 \%$ \\
Recurrence & $20(48 \%)$ & $15(79 \%)$ & $23(59 \%)$ \\
$\quad$ Local & $16(38 \%)$ & $8(42 \%) \dagger$ & $12(31 \%) \ddagger$ \\
Distant & $4(10 \%)$ & $7(37 \%)$ & $12(31 \%)$ \\
\hline
\end{tabular}

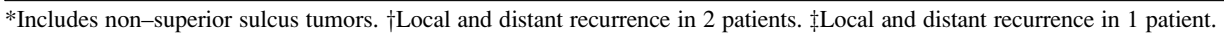

technique of resection was different in that vertebrectomy was performed by using an en bloc technique rather than the intralesional approach used in our study. Only 4 $(21 \%)$ patients underwent total vertebrectomy compared with $15(48 \%)$ patients in our series. Although median survival was excellent (24 months), 2-year survival was similar to that seen in our series ( $53 \%$ vs $47 \%$ ), but 5 -year survival was slightly inferior (14\% vs 27\%). Grunenwald and coworkers ${ }^{5}$ found that patients with negative margins after surgical intervention had better median and 2-year survival (34 months and $74 \%$, respectively), which is similar to what we have observed (39 months and 62\%, respectively).

A third study, by Fadel and associates, ${ }^{16}$ looked at en bloc hemivertebrectomies in 17 patients with superior sulcus lung cancer. Their study had a higher $\mathrm{R} 0$ resection rate than in our present series (76\% vs 56\%). However, the recurrence rates $(59 \%$ vs $59 \%)$ and 5-year survival rates (20\% vs $27 \%)$ were similar in both studies.

Despite using what might be thought a more oncologically sound approach of en bloc resection, rates of local and distant recurrence were similar between our series and that of Grunenwald and coworkers (Table 4). ${ }^{5}$ In addition, the technique of en bloc vertebrectomy was associated with a higher incidence of postoperative complications and longer hospital course than in our study. Furthermore, total vertebrectomy mandated use of a plastic brace for spine immobilization for up to 3 months postoperatively. Because of the frequent use of both posterior and anterior spine fixation and reconstruction of resected vertebral bodies at our institution, patients do not routinely require an external prosthesis.

In 1956, Chardack and MacCallum ${ }^{17}$ reported the 5-year survival of a patient who underwent surgical resection of a superior sulcus tumor followed by postoperative radiation.
It was not until 5 years later that Shaw and colleagues ${ }^{18}$ published their experience with preoperative radiation (30-35 Gy) and surgical resection in 18 patients who achieved good local control (despite no 5-year survivors). This landmark study has influenced management of superior sulcus tumors for more than 40 years. Despite no randomized data comparing preoperative with postoperative treatment, neoadjuvant chemoradiation has become the standard of care. ${ }^{19}$ Certainly, the encouraging results of Southwest Oncology Group Trial 9416, which reported 44\% 5-year survival with preoperative cisplatin/etoposide and radiation to $45 \mathrm{~Gy}$, argues in favor of the use of preoperative treatment for superior sulcus tumors. ${ }^{20}$ However, whether an induction approach is appropriate for tumors that invade the spine is uncertain. The majority of patients in Southwest Oncology Group Trial 9416 had T3 tumors, and of the $32(29 \%)$ patients with clinical T4 tumors, only $23(72 \%)$ underwent resection, most without vertebrectomy. Nineteen (59\%) patients with T4 disease had recurrence, and of these, 9 $(28 \%)$ had local failure, which is similar to the rate we observed in our study. The only predictive factor for longterm survival in Southwest Oncology Group Trial 9416 was a complete pathologic response at the time of the operation, which was achieved in $29 \%$ of patients.

Another prospective study of induction therapy with surgery for superior sulcus tumors was the Japan Clinical Oncology Group trial 9806, ${ }^{21}$ which reported 5-year survival of $56 \%$. These investigators found that complete pathologic response (present in 12 [16\%] patients), complete resection, and T3 tumor stage were associated with long-term survival. Of importance, only $11(55 \%)$ patients with T4 tumors underwent surgical resection, and of these, only 3 had vertebrectomy. 
A third study that evaluated induction therapy for superior sulcus tumors was that by Fischer and associates in 2008. ${ }^{22}$ Their study had 44 patients who underwent 2 cycles of cisplatin and etoposide with concurrent 45-Gy radiation. Mortality in this study was $5 \%$, an R0 resection was achieved in $89 \%$, and the recurrence rate was $36 \%$. Fiveyear survival for the entire group was $59 \%$. For patients with a complete response to induction therapy, a 5-year survival of $90 \%$ was achieved. For patients with no response to induction therapy, the 5-year survival was $12 \%$. They concluded that surgical resection after induction therapy was safe and could be achieved in patients who responded with good survival. However, this study had only 15 patients who had a portion of a vertebral body resected. This makes it difficult to interpret the results from this study when looking at patients who require vertebral body resection.

Although our numbers were small, we did not observe a difference in survival outcomes between patients receiving preoperative versus postoperative adjunctive therapy. In contrast with the studies above, of 16 patients who received preoperative treatment, only $1(6 \%)$ pathologic complete response was seen, and there were only $2(13 \%)$ patients with microscopic residual tumor after induction treatment. Furthermore, when compared with the reports by Bilsky and associates ${ }^{15}$ and Grunenwald and coworkers, ${ }^{5}$ in which use of preoperative treatment was favored, survival rates and patterns of recurrence in our study were similar. The data available therefore do not present a strong argument in favor of preoperative over postoperative regimens in patients with superior sulcus tumors with vertebral involvement.

Our philosophy with respect to adjunctive therapies in the management of these tumors has been in favor of immediate surgical resection, followed by postoperative radiation and chemotherapy. The rationale for this approach is as follows. First, in patients who receive preoperative radiation and who are subsequently found at the time of surgical intervention to have positive margins, additional postoperative radiation therapy is usually not feasible, and local tumor recurrence is virtually guaranteed. Second, immediate surgical intervention not only offers quicker and more durable palliation of pain than chemoradiation but also avoids the risk of tumor progression in chemotherapy and radiation-insensitive tumors. Third, there are reliable data regarding the survival benefit of postoperative chemotherapy for stage I to III lung cancer, whereas the data regarding use of preoperative chemotherapy is less convincing. Lastly, administration of high-dose radiation preoperatively can complicate surgical resection because of fibrosis and obliteration of normal tissue planes.

As with all retrospective studies, ours has several limitations. First, the patients in this study represent a group of patients who were considered to have tumors amenable to resection and who were physiologically able to withstand surgical intervention. This group might not be reflective of the entire population of patients with superior sulcus tumors and vertebral involvement. Second, the treatment period spanned 20 years, during which preoperative staging, adjunctive regimens, and surgical techniques have evolved. Although all of the resections were performed at a single institution, adjunctive treatment was often performed elsewhere, and this adds to treatment variation and inconsistency. Third, despite the fact that this series represents one of the largest series of patients with superior sulcus NSCLC and vertebral invasion, the sample size is still small.

Nevertheless, we believe that certain conclusions can be drawn from the data presented. We have confirmed the feasibility of concomitant lung and vertebral resection in selected patients with superior sulcus tumors and vertebral invasion. Long-term survival is possible and is most likely in patients who can undergo complete tumor resection and who do not have nodal disease. For this reason, we recommend routine preoperative evaluation of mediastinal nodes. Patients found to have lymph node metastasis preoperatively have a poor prognosis, and nonsurgical management should be considered for these patients. We believe that chemotherapy and radiation are important components of the treatment regimen for these patients, and for reasons discussed above, we favor postoperative rather than preoperative therapy, although there are few data to support one approach over the other. Although retrospective and based on small numbers of patients, the available data do not show a major difference in local recurrence or survival between en bloc versus intralesional approaches. Lastly, with the use of a multidisciplinary approach, vertebral involvement should not necessarily be a contraindication to resection in patients with superior sulcus tumors, and these patients deserve surgical consultation before development of a definitive treatment plan at specialized centers.

\section{References}

1. Komaki R, Roth JA, Walsh GL, Putnam JB Jr, Vaporciyan A, Lee JS, et al. Outcome predictors for 143 patients with superior sulcus tumors treated by multidisciplinary approach at the University of Texas MD Anderson Cancer Center. Int J Radiat Oncol Biol Phys. 2000;48:347-54.

2. Grunenwald D, Mazel C, Girard P, Berhiot G, Dromer C, Baldeyrou P. Total vertebrectomy of en bloc resection of lung cancer invading the spine. Ann Thorac Surg. 1996;61:723-6.

3. York JE, Walsh GL, Lang FF, Putnam JB, McCutcheon IE, Swisher SG, et al. Combined chest wall resection with vertebrectomy and spinal reconstruction for the treatment of Pancoast tumors. J Neurosurg. 1999;91:74-80.

4. Gandhi S, Walsh GL, Komaki R, Gokaslan ZL, Nesbitt JC, Putnam JB, et al. A multidisciplinary surgical approach to superior sulcus tumors with vertebral invasion. Ann Thorac Surg. 1999;68:1778-84.

5. Grunenwald D, Mazel C, Girard P, Veronesi G, Spaggiari L, Gossot D, et al. Radical en bloc resection for lung cancer invading the spine. J Thorac Cardiovasc Surg. 2002;123:271-9.

6. Koizumi K, Haraguchi S, Hirata T, Hirai K, Mikami I, Yamagishi S, et al. Surgical treatment of superior sulcus tumors. Surg Today. 2005;35:357-63.

7. Yokomise H, Gotoh M, Okamoto T, Yamamoto Y, Ishikawa S, Liu D, et al. En bloc partial vertebrectomy for lung cancer invading the spine after induction chemoradiotherapy. Eur J Cardiothorac Surg. 2007;31:788-90.

8. Cooper PR, Errico TJ, Martin R, Crawford B, DiBartolo T. A systematic approach to spinal reconstruction after anterior decompression for neoplastic disease of the thoracic and lumbar spine. Neurosurgery. 1993;32:1-8.

9. Errico TJ, Cooper PR. A new method of thoracic and lumbar body replacement for spinal tumors. Neurosurgery. 1993;32:678-81. 
10. de Perrot M, Rampersuad R. Anterior transclavicular approach to malignant tumors of the thoracic inlet: importance of the scapulothoracic articulation. J Thorac Cardiovasc Surg. 2007; 134:801-3.

11. Pancoast HK. Importance of careful roentgen-ray investigations of apical chest tumors. JAMA. 1924;83:1407-11.

12. Tobias JW. Sindrome apico-costo-vertebral doloroso por tumor apexiano: su valor diagnostico en el cancer primitvo pulmonary. Rev Med Latino Am. 1932;17:1522-56.

13. Dartevelle PG, Chapelier AR, Macchiarini P, Lenot B, Cerrina J, Ladurie FL, et al. Anterior transcervical-thoracic approach for radical resection of lung tumors invading the thoracic inlet. J Thorac Cardiovasc Surg. 1993;105:1025-34.

14. DeMeester TR, Albertucci M, Dawason PJ, Montner SM. Management of tumor adherent to the vertebral column. J Thorac Cardiovasc Surg. 1989;97:373-8.

15. Bilsky MH, Vitaz TW, Boland PJ, Bains MS, Rajaraman V, Rusch VW. Surgical treatment of superior sulcus tumors with spinal and brachial plexus involvement. J Neurosurg. 2002;97:301-9.

16. Fadel E, Missenard G, Chapelier A, Mussot S, Leroy-Ladurie F, Cerrina J, et al. En-Bloc resection of non-small cell lung cancer invading the thoracic inlet and intervetrebal foramina. J Thorac Cardiovasc Surg. 2002;123:676-85.

17. Chardack WM, McCallum JD. Pancoast tumor: five-year survival without recurrence or metastases following radical resection and postoperative irradiation. J Thorac Surg. 1956;31:535-42.

18. Shaw RR, Paulson DL, Kee JL Jr. Treatment of the superior sulcus tumor by irradiation followed by resection. Ann Surg. 1961;7:29-40.

19. Rusch VW, Parekh KR, Leon L, Venkatranam E, Bains MS, Downey RJ, et al. Factors determining outcome after surgical resection of T3 and T4 lung cancers of the superior sulcus. J Thorac Cardiovasc Surg. 2000;119:1147-53.

20. Rusch VW, Giroux DJ, Krault MJ, Crowley J, Hazuka M, Winton T. Induction chemoradiation and surgical resection for superior sulcus non-small-cell lung carcinomas: long term results of Southwest Oncology Group Trial 9416 (Intergroup Trial 0160). J Clin Oncol. 2007;25:313-8.

21. Kunitoh H, Kato H, Tsuboi M, Shibata T, Asamura H, Ichonose Y, et al. Phase II trial of preoperative chemoradiotherapy followed by surgical resection in patients with superior sulcus non-small-cell lung cancers: report of Japan Clinical Oncology Group Trial 9806. J Clin Oncol. 2008;26:644-9.

22. Fischer S, Darling G, Pierre AF, Sun A, Leighl N, Waddell TK, et al. Induction chemoradiation therapy followed by surgical resection for non-small cell lung cancer (NSCLC) invading the thoracic inlet. Eur J Cardiothorac Surg. 2008; $33: 1129-34$.

\section{Discussion}

Dr Marc de Perrot (Toronto, Ontario, Canada). Dr Bolton, that was a very nice presentation and I would like to congratulate you and your colleagues from MD Anderson for reporting on this very challenging group of patients.

Spinal resection for non-small cell lung cancer has now been reported by several groups with good results and is certainly the best option for a carefully well-selected group of patients.

In Toronto we have done about 25 hemivertebrectomy and a handful of total vertebrectomy for non-small cell lung cancer involving the spine. Although I don't want to spend too much time describing our results, I think it is important to emphasize that our approach has been different than the one from MD Anderson in the sense that we have used induction chemoradiation therapy for all of our patients and have completed the resection en bloc between the lung and the spine. The approach you have just presented has been primarily surgery with an intralesional resection of the tumor rather than an en bloc resection, as you mentioned. This difference probably results from the fact that you have been working with neurosurgeons, whereas we have been working with orthopedists primarily. However, I think when comparing the two approaches, it is important to make sure that you are comparing the same group of patients.

As you described, there are several types of spinal resections with different magnitudes of surgery that can be required for tumors invading the spine. Resection of the transverse process or wedge resection of the edge of the vertebral body that was mainly presented in your groups 1 and 2 are less extensive than the hemivertebrectomy or total vertebrectomy that you have included in group 3 . The spinal resection for group 1 and 2 can usually be done through the same incision as the lung resection and there is usually no need for any spinal reconstruction. When a hemivertebrectomy or total vertebrectomy is performed at the level of T1, it requires an anterior and a posterior approach in order to completely remove the tumor en bloc with the spine and to achieve adequate reconstruction of the spine.

In your presentation, you have included patients with involvement of the neuroforamen along with patients who had involvement of the transverse process in the same group. I don't think these two groups of patients are similar, at least in our experience. In our practice, patients with involvement of the neuroforamen would usually have an en bloc hemivertebrectomy with the lung resection in order to be able to section the nerve root inside the spinal canal and achieve complete resection. This would then be followed by spinal stabilization. Patients with involvement of the transverse process otherwise would have resection of the transverse process with the chest wall resection and would not require any type of spinal stabilization.

So my first question is, how many patients in your first group had involvement of the neuroforamen versus involvement of the transverse process and how many patients required spinal stabilization in that specific group of patients?

Dr Bolton. Thank you for your comments and question.

I think that your question is how many patients in group 1,8 patients, had neuroforamen involvement by itself or transverse process by itself. We did not break that group into a smaller group. There were only 8 of those. So I don't have the information of which ones had just neuroforamen involvement. I do know that none of those patients had to have a spinal stabilization for their resection.

Dr de Perrot. My second question relates to the surgical resection margins. You mentioned that $44 \%$ of your patients had a positive resection margin, which is higher than what has been reported in other series. This may be due to the fact that you have included only patients with superior sulcus tumors. However, considering that you do not attempt to do an en bloc resection but perform an intralesional resection, I'm wondering how you could interpret the surgical margin for patients who did not have en bloc resection.

Dr Bolton. Again, I believe your question is how do we assess the surgical margin in the operating room during the operation. With that, on the total vertebrectomies, we assess the margins by taking the soft tissues around the vertebral body that we have resected, and that can be done at the time of the operation. On patients with a partial vertebrectomy, obviously we can't send bone for a frozen section, and so we would use the high-speed drill to drill through to bone that appeared normal and then take samples of that and send that for a permanent section. So that's how we assessed our margins.

Dr de Perrot. So if the final specimen was negative, that was your negative margin?

Dr Bolton. But that would come back on a final pathologic specimen. We would not have that information during the operation. On the partial vertebrectomies, we would do it to grossly normal-appearing bone. 
Dr de Perrot. My last question relates to the group of patients who had total vertebrectomy. You mentioned 15 patients in that group, which is certainly the largest experience reported in the literature. Looking more specifically at that group of patients, you reported an $\mathrm{R} 0$ resection rate of about $50 \%$, with a local recurrence rate of about $40 \%$, and a 5-year survival of $22 \%$. Again, I think this is certainly the most difficult group of patients to manage because of the morbidity that the tumor by itself will create if it is not controlled locally and because of the complexity of performing a surgical resection in these patients. My last question is, among all 15 total vertebrectomies that you have done, were all these patients treated with a curative intent or were some of them treated to achieve primarily local control? Along the same line, you mentioned that 11 patients had a wedge resection rather than a more formal lobectomy, and I would be interested to know if these 11 patients were more specifically in that group of patients who had a total vertebrectomy.

Dr Bolton. We did not look at how many patients in the total vertebrectomy group had a wedge resection versus a lobectomy versus a pneumonectomy, so I can't give you that answer.

What was the rest of your question?

Dr de Perrot. I'm just wondering whether all these patients were operated on with a curative intent or were some of them undergoing surgery rather to achieve local control because of the morbidity of these tumors.

Dr Bolton. Yes, there were 4 patients in our study that we did a palliative resection on. Again, I don't know exactly where all 4 of those are. Some of those were in the total vertebrectomy group, but not all of them.

Dr de Perrot. Thank you.

Dr Bolton. Thank you for your questions.

Dr Frank C. Detterbeck (New Haven, Conn). How do you preoperatively stage the patients, nodal staging?

Dr Bolton. How many were preoperatively staged, is that the question?

Dr Detterbeck. Well, how do you stage them? Do you do mediastinoscopy? Do you do PET? What do you do?

Dr Bolton. This was over a long period of time, and so the optimal staging for mediastinal.

Dr Detterbeck. What do you think it should be now, given your experience?

Dr Bolton. Well, given our experience, with the fact that we found no patients with a positive node either in stations 1,2 , or 3 that lived more than 23 months, I think that if you're going to do surgery for this group of patients, you need to adequately stage the mediastinum with mediastinoscopy or EBUS. With EBUS you have the ability to maybe stage more than just the mediastinum and stage levels 10 and 11 lymph nodes, and so that would be an additional benefit to use EBUS in this patient population.

Dr Detterbeck. The second question has to do with the high rate of incomplete resection. What do you think we can do to improve that? Certainly preoperative chemoradiotherapy in Pancoast tumors in general has resulted in a much higher rate of complete resection.
Do you think that that is the answer? The other related question is selection. What criteria do you have to make you say this patient is not resectable; there is either too much vertebral body involvement, there's involvement of the dura, there's some other aspect of what contributes to a positive margin that makes that patient not a good candidate?

Dr Bolton. Thank you for your question.

Looking at the assessment of whether or not we will be able to get the patient resected, there have been a couple of studies that have looked at using preoperative imaging, such as MRI, to sort of help determine whether or not you could be more successful. Unfortunately, those studies did not show a difference on any of their definitions of how much vertebral body involvement was found preoperatively. I don't think that we have a good imaging modality to help us with that. Certainly if you had a patient with multiple vertebral bodies destroyed by tumor, that would be an easy patient to say that you could not do it on, but otherwise I don't think the imaging available currently can tell us that on the minor points.

Dr Dominique Grunenwald (Paris, France). I was extremely anxious to discover the late results of the MDA's multidisciplinary approach, since we presented at this meeting here in San Diego in year 2001 the initial part of our series of en bloc resection for nonsmall cell lung cancer attached to the spine. Interestingly, our updated data, despite the difference in our respective approaches, show similar results now. The differences are in the surgical procedures, as we performed an en bloc resection in all cases. Secondly, the extent of resection is quite different, because our series includes a large majority of partial resections, of hemivertebrectomies (28 among 34 patients). Thirdly, the complete resection rate that we achieved was $90 \%$ compared to your $56 \%$ of complete resections. Nevertheless, results in terms of long-term survival, 5-year survival, are absolutely the same, because we achieved a $24 \% 5$ year survival now compared to your $27 \%$ survival. But we didn't include the patients with only a transverse or foraminal involvement. So our results and these comments confirm your conclusions, Dr Bolton. Congratulations.

Dr Bolton. Thank you very much, sir.

Dr Alain Chapelier (Paris, France). Congratulations on this impressive series of very difficult patients requiring demanding surgical strategies. I have a comment and a short question. Earlier this year, Philippe Dartevelle and colleagues reported the long-term results of surgery of superior sulcus tumors with $28 \%$ 5-year survival in the group of patients with en bloc resection, partial vertebrectomy. However, this tumor was only extending into the intraforaminal process. So my question is, considering the poor survival of the subset of patients with positive margins, wouldn't you be reluctant to consider such extended surgery if you don't have a strong probability of achieving a complete resection?

Dr Bolton. Thank you for your question.

Looking at our data, I would say that it would be much better to be able to get a negative margin on your resection. So, yes, we would much prefer to be able to get a negative margin. 